Rodrigo Arturo Tardio Flores. ${ }^{1}$ (D)

Luis Alejandro Lafuente Lafuente. ${ }^{2}$ (iD

Alejandra Estefany Melgarejo Vidal. ${ }^{2}$ (iD

Ericka Ayde Mamani

Salas. ${ }^{2}$ iD

Lizeth Chacon Paco. ${ }^{2}$

Dilean Elfy Ayaviri. ${ }^{3}$ (DD

Correspondencia a:

1 Medico anestesiólogo hospital de segundo nive

"San Juan de Dios" del municipio de Cliza.

Cochabamba - Bolivia

2 Centro de investigación medicina UNITEPC "CIMU".

Cochabamba - Bolivia

3 Jefa del departamento de patología de medicina universidad técnica privada cosmos UNITEPC.

Centro de investigación de salud pública UNITEPC. "CISPU".

Cochabamba - Bolivia

Email de contacto:

rodrigotf79@hotmail.com naelidayaviri@gmail.com

Procedencia y arbitraje: No comisionado, sometido a arbitraje externo

Recibido para publicación: 18 de mayo del 2019 Aceptado para publicación: 25 de junio del 2019

Citar como:

$\mathrm{Re} \mathrm{Ci} \mathrm{Sa}$ UNI

2019;6(2):14-19

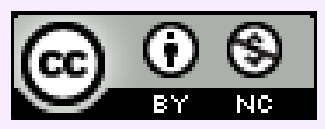

Esta obra está bajo una Licencia Creative Commons Atribución-NoComercia 4.0 Internacional.

\section{Protozoariasis intestinal en edad pediátrica}

\section{Pediatric intestinal protozoariasis in children}

\section{Protozoose intestinal em idade pediátrica}

\section{Resumen}

Introducción: La protozoariasis tiene una alta prevalencia en diferentes regiones de nuestro país y es uno de los principales problemas, por su alta prevalencia y elevados índices de transmisibilidad, el objetivo del presente trabajo es determinar las características de la protozoariasis intestinal en edad pediátrica. Metodología: Se realizó un estudio descriptivo, transversal y retrospectivo, se revisó los resultados de estudios de laboratorio de la gestión 2017 y 2018 de pacientes que acudieron al hospital San Juan de Dios del municipio de Cliza Cochabamba, Bolivia. Resultados: La frecuencia de protozoariasis fue $56.8 \%$, según el sexo $52,2 \%$ mujeres y $47,8 \%$ varones, en el grupo etario de niños menores de 1 año 27\%, 1 a 5 58.4\%, 5 a $1010.3 \%, 10$ a 12 $3 \%$ y 12 a $151.3 \%$. La frecuencia del tipo de protozoario es Entamoeba histolytica 78.6\%, Giardia lamblia 17.6\%, Chilomastix mesnili $1.8 \%$, Blastocystis hominis $1.2 \%$ y Endolimax nana $0.7 \%$. Discusión: Se observa una disminución en la prevalencia de estos microorganismos, considerando la mejora de los servicios básicos en la zona y educación sanitaria, siendo frecuente en el sexo femenino, y el grupo etario que presento mayor parasitosis es de 1 a 5 años, siendo la Entamoeba histolytica la de mayor presencia y no existe relación en la frecuencia de protozoariasis intestinal en relación con otros trabajos de la región.

Palabras claves: Parasitología, Infecciones por Protozoos, Pediatría, Niño, Prevalencia.

\section{Summary}

Introduction:The protozoariasis has a high prevalence in different regions of our country and it is one of the main problems, due to its high prevalence and high transmissibility indexes, the present work's objective is to determine the intestinal protozoariasis characteristics in pediatric age. Methodology: A descriptive, cross-sectional and retrospective study was conducted, the 2017 and 2018 laboratory studies results from patients who visited the San Juan de Dios hospital in the municipality of Cliza Cochabamba, Bolivia were reviewed. Results: The protozoariasis frequency was $56.8 \%$, according to sex $52.2 \%$ women and $47.8 \%$ men, in the children age group under 1 year $27 \%$, from 1 to 5 years $58.4 \%$, from 5 to 10 years $10.3 \%$, from 10 to 12 years 3\% and from 12 to 15 years $1.3 \%$. The frequency of the type of protozoan is Entamoeba histolytica 78.6\%, Giardia lamblia 17.6\%, Chilomastix mesnili 1.8\%, Blastocystis hominis 1.2\% and Endolimax nana 
$0.7 \%$. Discussion: There is a decrease in the prevalence of these microorganisms, considering the improvement of basic services in the area and health education, being more frequent in females, and the age group that presented the highest parasitosis is from 1 to 5 years, Being Entamoeba histolytica the one with the greatest presence and there is no relationship in the protozoariasis intestinal frequency in relation to other works in the region.

Keywords: Parasitology, Protozoan Infections, Pediatrics, Child, Prevalence

\section{Resumo}

Introdução: As parasitosis possuem alta prevalência em diferentes regiões do país,e é um dos principais problemas,por sua alta prevalência e elevados índices de transmissibilidade, $\mathrm{O}$ objetivo do presente trabalho é determinar as características da doencas parasitárias em idade pediátrica. Metodologia: Foi realizado um estudo descritivo, transversal e retrospectivo; sendo revisados os resultados de estudos laboratoriais dos pacientes de 2017 a 2018 que visitaram o Hospital San Juan de Dios, no município de Cliza Cochabamba, Bolívia. Resultados: A frequência de doencas parasitárias foi de $56,8 \%$, segundo o sexo, $52,2 \%$ de mulheres e $47,8 \%$ de homens, na faixa etária de crianças menores de 1 ano 27\%, 1 a 5 58,4\%, 5 a 10 10,3\%, 10 a $123 \%$ e 12 a 15 1,3\%. A frequência do tipo de protozoário é Entamoeba histolytica 78,6\%, Giardia lamblia 17,6\%, Chilomastix mesnili 1,8\%, Blastocystis hominis 1,2\% e Endolimax nana 0,7\%. Discussão: Há uma diminuição na prevalência desses microrganismos, considerando a melhoria dos serviços básicos na área e educação em saúde, sendo mais frequente no sexo feminino, e a faixa etária que apresentou a frequencia em parasitoses é de 1 a 5 anos, Entamoeba histolytica é a que apresenta maior presença e não há relação na frequência de protozoários intestinais em relação a outros trabalhos na região.

Palavras chave: Parasitologia, Infecções por Protozoários, Pediatria, Criança, Prevalência,

\section{Introducción}

La protozoariasis intestinal tiene una distribución global y presenta altas tasas de prevalencias en diferentes regiones. Por lo general no tienen una elevada mortalidad, pero de igual manera ocasionan un impacto negativo en la salud y economía de sus huéspedes (1).

En nuestro país es uno de los principales problemas de salud pública, por su alta prevalencia y elevados índices de transmisibilidad, la mayoría de estos parásitos se caracterizan por presentar resistencia a las diferentes condiciones medioambientales, por lo que permite tener supervivencia a factores físicos, químicos y tratamientos farmacológicos $(2,3)$.

Dentro del grupo de protozoarios intestinales se encuentran: Entamoeba histolytica, Giardia lamblia, Balantidium coli, Entamoeba hartmanni, lodamoeba buetschlii, Dientamoeba fragilis, Endolimax nana, Cryptosporidium parvum, Cyclospora cayetanensis, Isospora belli, Chilomastix mesnili y Blastocystis hominis (3).

Los hábitos y el comportamiento humano tienen mucha importancia para la transmisión de protozoarios, por dicha razón es necesario implementar medidas que modifi- 
quen los hábitos no saludables y los comportamientos inadecuados (4).

Se observa que la población de las zonas rurales y periurbanas, poseen viviendas precarias que carecen de servicios básicos sanitarios. La falta de acceso a agua potable, servicio de alcantarillado, bajo nivel de educación y además de las características de trabajo agropecuario son factores que los torna vulnerables a contraer parasitosis intestinales.

Existe contaminación con aguas servidas no tratadas en ríos y vertientes, y esta es usada para consumo humano y agropecuario, en este sentido podemos suponer que contienen parásitos, los cuales desencadenan enfermedades por contaminación alimentaria y diseminación parasitaria importante. Agregado a estos factores se observa los inadecuados hábitos de higiene y manipulación de alimentos $(5,6)$.

Los niños al iniciar la locomoción (arrastrarse, gatear, sentarse y caminar) y la asistencia a centros infantiles aumentan la interacción con el medio ambiente incrementando el riesgo de contraer enfermedades parasitarias $(7,8)$.

El objetivo del presente trabajo es determinar las características de la protozoariasis intestinal en edad pediátrica.

\section{Metodología}

Se realizó un estudio descriptivo, transversal y retrospectivo, revisando los cuadernos de resultados de estudios de laboratorio "coproparasitológicos" de la gestión 2017 y 2018 de pacientes en edad pediátrica "menores de 15 años", que acudieron al hospital de segundo nivel "San Juan de Dios" del municipio de Cliza de la provincia Germán Jordán del departamento de Cochabamba, Bolivia. Todo esto con autorización del personal responsable del departamento de laboratorio de análisis clínico del hospital.

La muestra estuvo conformada por 748 resultados de estudios coproparasitológicos, que cumplieron con los criterios de inclusión establecidos en el protocolo.

La técnica y el instrumento utilizado fue, la observación y el registro de datos en planilla conformada por las distintas variables del estudio, posterior al cual se introdujo en el programa IBM SPSS para análisis e interpretación.

\section{Resultados}

De los 748 resultados de estudios coproparasitológicos hechos en el laboratorio de análisis clínico del hospital de Cliza muestran: $56.8 \%(n=425)$ presentaban algún tipo de protozoario intestinal y $43.2 \%(n=323)$ no.

La frecuencia de protozoariasis intestinal en edad pediátrica según el sexo fue: $52,2 \%$ mujeres y el $47,8 \%$ varones.

Según los grupos etarios la frecuencia de protozoariasis intestinal fue: Niños menores de 1 año 27\%, 1 a 5 años el 58.4\%, 5 a 10 años 10.3\%, 10 a 12 años 3\% y 12 a 15 años $1.3 \%$. Ver cuadro 1.

En relación con la frecuencia del tipo de protozoario intestinal se observa: Entamoeba histolytica $78.6 \%$, Giardia lamblia 17.6\%, Chilomastix mesnili 1.8\%, Blastocystis hominis 1.2\%, Endolimax nana 0.7\%. Ver cuadro 1.

Relacionando los grupos etarios y la frecuencia de protozoarios intestinales se en- 
contró menores de 1 año; 82 niños tienen Entamoeba histolytica, 32 Giardia lamblia, 1 Chilomastix mesnili. De 1 a 5 años; 204 Entamoeba histolytica, 35 Giardia lamblia, 2 Blastocystis hominis, 6 Chilomastix mesnili, 1 Endolimax nana. De 5 a 10 años; 33 Entamoeba histolytica, 6 Giardia lamblia, 2 Blastocystis hominis, 1 Chilomastix mesnili, 2 Endolimax nana. De 10 a 12 años; 10 Entamoeba histolytica, 1 Giardia lamblia, 1 Blastocystis hominis. De 12 a 15 años; 5 Entamoeba histolytica, 1 Giardia lamblia. Ver cuadro 1.

Cuadro 1. Relación de la edad con la frecuencia de protozoarios intestinales

\begin{tabular}{|c|c|c|c|c|c|c|}
\hline \multirow{2}{*}{ Edad } & \multicolumn{6}{|c|}{ Protozoarios } \\
\hline & $\begin{array}{l}\text { Entamoeba } \\
\text { histolytica }\end{array}$ & $\begin{array}{l}\text { Giardia } \\
\text { lamblia }\end{array}$ & $\begin{array}{l}\text { Blastocystis } \\
\text { Hominis }\end{array}$ & \begin{tabular}{|l|}
$\begin{array}{l}\text { Chilomastix } \\
\text { mesnili }\end{array}$ \\
\end{tabular} & $\begin{array}{l}\text { Endolimax } \\
\text { Nana }\end{array}$ & Total \\
\hline \multirow{2}{*}{$<1$} & & 32 & \multirow{2}{*}{0} & 1 & \multirow{2}{*}{0} & 115 \\
\hline & $19,3 \%$ & $7,5 \%$ & & $0,2 \%$ & & $27,1 \%$ \\
\hline \multirow{2}{*}{$1-5$} & 204 & 35 & 2 & 6 & 1 & 248 \\
\hline & $48 \%$ & $8,2 \%$ & $0,5 \%$ & $1,4 \%$ & $0,2 \%$ & $58,3 \%$ \\
\hline \multirow{2}{*}{$5-10$} & 33 & 6 & 2 & 1 & 2 & 44 \\
\hline & $7,8 \%$ & $1,4 \%$ & $0,5 \%$ & $0,2 \%$ & $0,5 \%$ & $10,4 \%$ \\
\hline \multirow{2}{*}{$10-12$} & 10 & 1 & 1 & \multirow{2}{*}{0} & \multirow{2}{*}{0} & 12 \\
\hline & $2,3 \%$ & $0,2 \%$ & $0,2 \%$ & & & $2,8 \%$ \\
\hline \multirow{2}{*}{$12-15$} & 5 & 1 & \multirow[b]{2}{*}{0} & \multirow[b]{2}{*}{0} & \multirow[b]{2}{*}{0} & 6 \\
\hline & $1,2 \%$ & $0,2 \%$ & & & & $1,4 \%$ \\
\hline \multirow{2}{*}{ Total } & 334 & 75 & 5 & 8 & 3 & 425 \\
\hline & $78,6 \%$ & $17,6 \%$ & $1,2 \%$ & $1,8 \%$ & $0.7 \%$ & $100 \%$ \\
\hline
\end{tabular}

Fuente: Propia del autor.

\section{Discusión}

Inicialmente el estudio se comparará con otros realizados en el mismo municipio de Cliza el 2004 y del municipio de Sacaba el 2016, los cuales se toman en cuenta por las características geográficas y socio culturales.

La frecuencia de protozoariasis intestinal en este trabajo fue del $56.8 \%$, en relación con otros estudios que muestran un $75.7 \%$ y $87 \%$, se observa una disminución en la prevalencia de estos microorganismos, considerando la mejora de los servicios básicos en la zona y educación sanitaria emprendido por el Hospital San Juan de Dios de Cliza $(10,11)$.

En la variable relacionado al sexo y la presencia de protozoarios, este estudio mostró que $52,2 \%$ eran mujeres y el $47,8 \%$ varones, con relación a otros estudios, $52 \%$ mujeres y $48 \%$ varones, existe una relación en este acápite siendo más frecuente en el sexo femenino (11).

Relacionando la edad con la frecuencia de protozoarios intestinales tenemos niños menores de 1 año $27 \%$ y otros estudios como el de Sacaba muestran $28 \%$, se observa que las prevalencias son las mismas en este grupo etario. De 1 a 5 años es $58.4 \%$, otros estudios muestran valores mayores al $72 \%$, existiendo una diferencia marcada en este grupo etario, aunque en todos los trabajos es el grupo más afectado $(10,11)$. En los otros grupos etarios no existe diferencia en la presencia de estos microorga- 
nismos (12).

Para relacionar el tipo de protozoario intestinal encontrado se comparará con otros estudios hechos en el departamento de Cochabamba, para esto se elaboró la siguiente tabla de comparaciones $(9,10,2,11,13)$ : ver tabla 2 .

Tabla 2. Comparación en porcentaje de la frecuencia de protozoarios intestinales en diferentes estudios

\begin{tabular}{|c|l|c|c|c|c|}
\hline Año: & Estudio: & $\begin{array}{l}\text { Entamoeba } \\
\text { histolytica }\end{array}$ & $\begin{array}{l}\text { Giardia } \\
\text { lamblia }\end{array}$ & $\begin{array}{l}\text { Blastocystis } \\
\text { hominis }\end{array}$ & $\begin{array}{l}\text { Chilomastix } \\
\text { mesnili }\end{array}$ \\
\hline 2019 & $\begin{array}{l}\text { Protozoarios intestinales } \\
\text { en edad pediátrica. }\end{array}$ & $78,6 \%$ & $17,6 \%$ & $1,2 \%$ & $1,9 \%$ \\
\hline 2016 & $\begin{array}{l}\text { Prevalencia de entero- } \\
\text { parasitosis en niños que } \\
\text { recibieron atención en el } \\
\text { hospital México, municipio } \\
\text { de Sacaba }\end{array}$ & $38 \%$ & $37 \%$ & $5 \%$ & $0 \%$ \\
\hline $\begin{array}{l}\text { Prevalencia de enteropa- } \\
\text { rasitosis en niños menores } \\
\text { de 12 años que asisten a } \\
\text { la Guardería Niño de Praga } \\
\text { de la localidad de Tiquipa- } \\
\text { ya }\end{array}$ & $16 \%$ & $37 \%$ & $55 \%$ & $11 \%$ \\
\hline 2012 & $\begin{array}{l}\text { Estudio descriptivo de las } \\
\text { enteroparasitosis en pa- } \\
\text { cientes del hospital mater- } \\
\text { no infantil German Urquidi }\end{array}$ & $37,2 \%$ & $37,5 \%$ & $0 \%$ & $0 \%$ \\
\hline 2012 & $\begin{array}{l}\text { Relación entre la escolari- } \\
\text { dad y la incidencia de en- } \\
\text { teroparasitosis infantil en la } \\
\text { ciudad de Quillacollo }\end{array}$ & $39,37 \%$ & $22 \%$ & $0 \%$ & $3,93 \%$ \\
\hline 2004 & $\begin{array}{l}\text { Enteroparasitosis en pobla- } \\
\text { ción escolar de Ucureña }\end{array}$ & $33,3 \%$ & $42 \%$ & $0 \%$ & $0 \%$ \\
\hline
\end{tabular}

Fuente: propia de los autores.

Se observa que no existe relación en la frecuencia de protozoariasis intestinales en relación con otros trabajos de la región, y se tiene que tener en cuenta que estos trabajos incluyeron a la totalidad de enteroparásitos y nuestro estudio consideró únicamente al grupo de protozoarios.

En conclusión, se encontró una prevalencia elevada de protozoarios intestinales en edad pediátrica atendidos en el Hospital San Juan de Dios de Cliza, siendo la Entamoeba histolytica el protozoario más frecuente, además, citar que contradice la prevalencia elevada de este parasito en relación a la Giardia lamblia. Esto abre las puertas para realizar nuevas investigaciones relativo al problema.

\section{Conflictos de Intereses}

Los autores declaramos no tener conflictos de interés para el presente estudio.

\section{Referencias bibliográficas}

1. Organización Mundial de la Salud. Prevención y control de las infecciones parasitarias intestinales. Un Informe de un Comité de Expertos de la OMS; 1987; Ginebra. p. 7. 
2. Mamani-Ortiz, Y, Rojas-Salazar EG, Choque-Ontiveros, MC, Caero-Suarez, RI. Relación entre la escolaridad y la incidencia de enteroparasitosis infantil en la ciudad de Quillacollo. Rev Méd-Cient “Luz Vida”. 2012; 3 (1): 31-35.

3. Solarte $\mathrm{Y}$, Peña M, Madera C. Transmisión de protozoarios patógenos a través del agua para consumo humano. Colomb Med 2006; 37 (1): 74-82.

4. Fos Claver S, Vendrell Blay E. Minardi Mitre R, Morales Suárez-Varela MM, Y Llopis González A. Enfermedades parasitarias de origen alimentario más frecuentes en España: incidencia y comparación con las de origen vírico y bacteriano. Ars Pharmaceutica. 2000; 41(3): 293-305.

5. Botero D, Restrepo M. Parasitosis Humanas. $5^{\circ}$ ed. Medellin, Colombia: Corporación para Investigaciones Biológicas - CIB; 2012.

6. Atias A. Parasitología Médica. 1 ed. Santiago - Chile: Mediterráneo; 2011.

7. Rojas DCL, Álvarez ID, Martínez DR, Cabrera DM, Cordoví TR, Kourí DCG. Segunda encuesta nacional de infecciones parasitarias intestinales en Cuba. Rev cubana Med Trop. 2012; 64(1): 15-21

8. Joyce T, Mcguigan KG, Elmore MM, Conroy RM. Prevalence of enteropathogens in stools of rural Maasai children under five years of age in the Maasailand region of Kenyan Rift Valley. East Afr Med.1996; 73(1): 59-62.

9. Rojas Salazar FM, Gómez Quispe HF, Salomon Nuñez del Prado Y, Céspedes Vargas J, Rojas Cabrera E, Zambrana Lizarazu J. Estudio descriptivo de las enteroparasitosis en pacientes del hospital materno infantil German Urquidi Cochabamba-Bolivia: Desde enero a diciembre del 2001. Rev Científica Cienc Med. 2003; 4: 53-56.

10. Rojas Terrazas LF. Enteroparasitosis en población escolar de Ucureña. 1 ed. Cochabamba: El artesanal; 2004

11. Angulo R, Cáceres M, Moreira E, Oliveira E, Do Vale J, de Melo Strelow S. Prevalencia de enteroparasitosis en niños que recibieron atención en el hospital México, municipio de Sacaba. Revista científica de salud UNITEPC. 2016; 1(2):15-23

12. Morales Del Pino J R. Parasitosis intestinal en preescolares y escolares atendidos en el centro médico EsSalud de Celendin, Cajamarca. Horiz. Med. [Internet]. 2016 Jul [citado 2019 Marzo 30]; 16(3): 35-42. Disponible en: http://www.scielo.org.pe/ scielo.php?script=sci_arttext\&pid=S1727-558X2016000300006\&Ing=es.

13. Villarroel García M, Hidalgo Filipovich R, Rojas Vásquez S, Martinez Eid G, Gómez Ugarte M, Escalera D, et al. Prevalencia de enteroparasitosis en niños menores de 12 años que asisten a la Guardería Niño de Praga de la localidad de Tiquipaya de la ciudad de Cochabamba, Bolivia, durante el semestre II/2015. Revista de Investigación e Información en Salud. 2017; 12(29): 24-30 